Available online at GSC Online Press Directory

GSC Biological and Pharmaceutical Sciences

e-ISSN: 2581-3250, CODEN (USA): GBPSC2

Journal homepage: https://www.gsconlinepress.com/journals/gscbps

(REVIEW ARTICLE)

\title{
Dengue fever: Causes, complications, and vaccine strategies - A review
}

\author{
Oviya S, Kaviya S and Udhaya S \\ Department of Microbiology, Annamalai University, Chidambaram, India.
}

Publication history: Received on 13 February 2019; revised on 28 February 2019; accepted on 04 March 2019

Article DOI: https://doi.org/10.30574/gscbps.2019.6.3.0024

\begin{abstract}
Dengue is an extremely endemic infectious disease of the tropical countries and is rapidly becoming a global burden. It is caused by any of the four serotypes of dengue virus and is transmitted within humans through female Aedes mosquitoes. Dengue disease varies from mild fever to severe conditions of dengue hemorrhagic fever and shock syndrome. Globalization, increased air travel, and unplanned urbanization have led to increase in the rate of infection and helped dengue to expand its geographic and demographic distribution. Dengue vaccine development has been a challenging task due to the existence of four antigenically distinct dengue virus serotypes, each capable of eliciting crossreactive and disease-enhancing antibody response against the remaining three serotypes. Recently, Sanofi Pasteur's chimeric live-attenuated dengue vaccine candidate has been approved in Mexico, Brazil, and Philippines for usage in adults between 9 and 45 years of age. The impact of its limited application to the public health system needs to be evaluated. Simultaneously, the restricted application of this vaccine candidate warrants continued efforts in developing a dengue vaccine candidate which is additionally efficacious for infants and native individuals. In this context, alternative strategies of developing a designed vaccine candidate which does not allow production of enhancing antibodies should be explored, as it may expand the umbrella of efficacy to include infants and native individuals.
\end{abstract}

Keywords: Dengue; Dengue causes; Complications; Vaccine

\section{Introduction}

Dengue is a communicable disease caused by any of the four dengue virus divergent serotypes: DENVs 1-4. It is a mosquito-borne disease and is primarily transmitted to humans by the female Aedes mosquito. The disease is mainly focused in tropical and subtropical areas, putting nearly a third of the human population, worldwide, at risk of infection [1]. Infection with DENV outcomes in different degrees of pathological conditions, ranging from mild asymptomatic dengue fever (DF) to severe dengue hemorrhagic fever (DHF) and dengue shock syndrome (DSS) which may turn fatal [2]. A dramatic universal expansion of the DENV has transpired due to rapid urbanization, increase in international travel, lack of effective mosquito control measures, and globalization [3].

Dengue has developed one of the most widespread reemerging mosquito-borne diseases globally. Incidence of dengue has increased 30-fold in last five decades [4]. Currently, dengue is endemic to 128 countries, mostly developing nations, posing a risk to approximately 3.97 billion people annually. A recent dengue distribution model has estimated 390 million dengue infections annually, out of which 96 million cases occurred apparently [5]. The Indian subcontinent is the epicenter of dengue [6] with cases being heavily underestimated [7]. Thus, there is an urgent need of improvement in serosurveillance to enable the authorities to prepare adequately for an outbreak.

\footnotetext{
${ }^{*}$ Corresponding author

E-mail address: manimicroscien@gmail.com
} 


\section{Epidemiology}

Dengue has become one of the most widespread recurring mosquito-borne diseases worldwide. Prevalence of dengue has increased 30-fold in last five decades [8]. Currently, dengue is endemic to 128 countries, mostly developing nations, posing a risk to approximately 3.97 billion people annually. A recent dengue distribution model has estimated 390 million dengue infections annually, out of which 96 million cases occurred apparently $[9,10]$. The Indian subcontinent is the epicenter of dengue [11] with cases being heavily undervalued [12]. Thus, there is an urgent need of improvement in serosurveillance to enable the authorities to prepare adequately for an outbreak.

\section{Route}

Dengue viruses are transmitted in humans by female Aedes (Ae.) mosquitoes of the subgenus Stegomyia. Ae. aegypti has been the most important epidemic vector in the tropical and subtropical regions. Other species such as Ae. albopictus, Ae. polynesiensis, member of Ae. scutellaris

\section{Dengue virus}

Dengue goes to the Flaviviridae family, which includes the West Nile and Yellow fever viruses (Westaway 1997) The dengue viral genomes consist of a positive single-stranded RNA encoding ten proteins; capsid (C), membrane protein (M), envelope protein (E), and seven nonstructural proteins, NS1, NS2A, NS2B, NS3, NS4A, NS4B and NS5 is a large multifunctional protein with a C-terminal RNA-dependent-RNA polymerase domain that is required for viral replication [13] and an N-terminal methyltransferase domain required for RNA capping.

The E protein is the major exposed antigen of the dengue virion, antibodies against which provide immunity during natural infection. The E proteins of the four DENV serotypes have 60-70\% amino acid similarity and are glycosylated at Asn-67 (unique to dengue) and Asn-153. These residues have been found to play important roles in the receptor attachment and viral entry into the cell. The E protein consists of a transmembrane region and an ectodomain which is divided into three structural/functional domains [14] (Fig. 1)

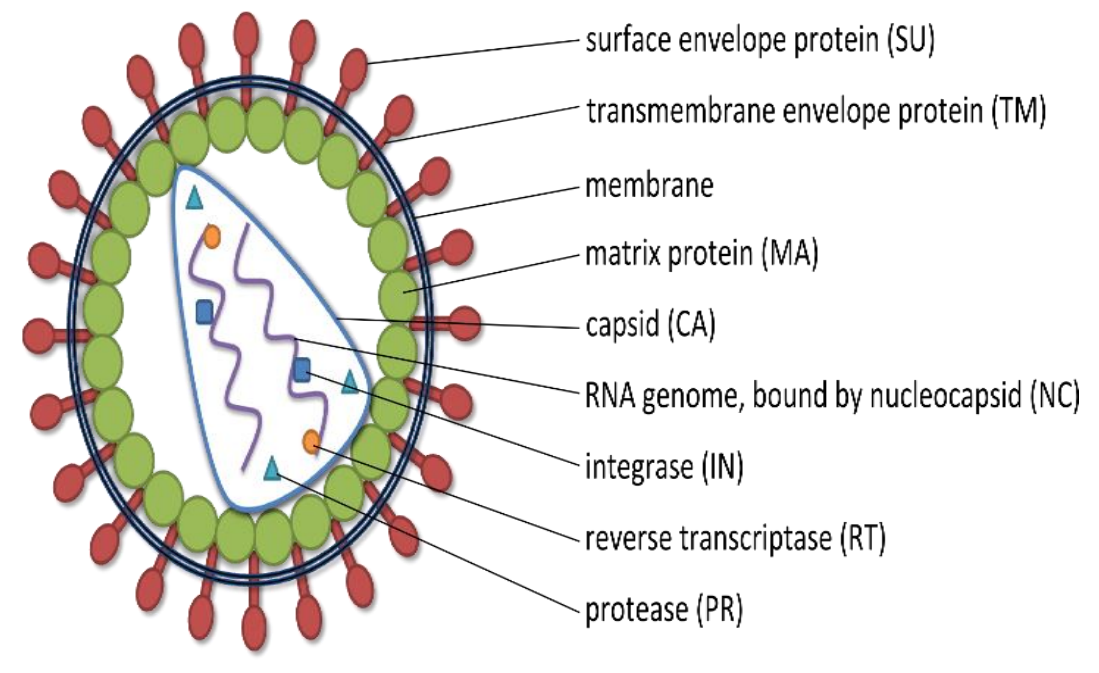

Figure 1 Structure of dengue virus

Capsid binds and stabilizes viral RNA. Premembrane / membrane i) Pr peptide functions as cap that protects the fusion peptide on $\mathrm{E}$, thus preventing premature fusion (ii) $\mathrm{M}$ forms ion channel. Envelope (E) (iii) Recognition and binding to the host cell Involved in uncoating of virus by enabling fusion of viral and endosomal membrane.

\section{Dengue causes}

The range of clinical sickness may extend from asymptomatic disease to a broad range of syndromes with severe clinical signs. Symptomatic infection may range from mild debilitating DF to life threatening DHF and DSS due to plasma outflow in DHF patients. These three conditions likely represent progressively severe stages of a continuous dengue disease spectrum [15]. They depend on traditional WHO classification case definitions and continue to be recognized in many 
regions of the world despite the introduction of a new classification system. The new classification based on a single parameter allows better case capture [16] but is not compatible with restricted health care facilities in endemic regions, especially during outbreaks [17].

DF is a self- restricting fever, lasting usually for 5-7 days. It is sometimes unbearable during the acute illness stage. The clinical features of DF vary according to the age of the patient. The children and young children may have undifferentiated febrile sickness with maculopapular rash. The older children and adults may have mild fevered syndrome or severe disease with high fever (usually biphasic), severe headache, retroorbital pain, myalgia, arthralgia, nausea, vomiting, and petechiae. Leukopenia and thrombocytopenia are usually observed in all ages. In some cases, DF may accompany bleeding complication such as gingival bleeding, epistaxis, gastrointestinal bleeding, haematuria, and menorrhagia (in case of women) [15].

DHF is described by symptoms of DF along with thrombocytopenia, hemorrhagic manifestations, and plasma spillage. A positive tourniquet test may be suggestive of DHF; however, this is being debated now due to its low sensitivity/specificity. Plasma leakage determines disease severity in DHF. It is also the most important difference between DHF and DF. Depending on disease severity and clinical manifestations, DHF is divided into four grades I to IV, with grade IV being the most severe. Numerous patients also have fine petechiae scattered on the extremities, axillae, face, and soft palate, usually seen in the febrile period. The critical phase is usually reached at the end of febrile illness, marked by rapid decrease in temperature and often accompanied by circulatory disturbances including plasma leakage, hemoconcentration, and thrombocytopenia.

In seroius cases, with critical plasma loss, DSS results and may be life threatening if not treated properly. DSS is categorized by a rapid, weak pulse with narrowing pulse pressure ( $<20 \mathrm{~mm}$ of $\mathrm{Hg}$ ), cold clammy skin, and restlessness. The patient may die within 12-24 h of going into shock or recover rapidly with volume replacement therapy.

\section{Dengue vaccine strategies}

In spite of the current difficulties for a perfect dengue immunization, improvement of dengue antibody applicants has advanced throughout the most recent decade and a portion of these have entered clinical preliminaries in both endemic and nonendemic territories.

\subsection{Replicating viral vaccines}

These include live-attenuated viruses (LAV) that are created by reducing the virulence of a pathogen without compromising its viability. Current methods of producing live-attenuated viruses for dengue vaccines include attenuation by serial passage in cell lines and targeted mutagenesis and by constructing chimeric vaccine viruses:

Advantages: robust, lasting, and broad immunity andlower production cost.

Disadvantages: difficulty in attenuation, genetic instability, possibility of reversion, and interference in the case of multicomponent LAV vaccines.

\subsection{Chimeric dengue vaccine}

Chimeric dengue immunizations have been planned using two methods: (i) with another attenuated flavivirus and (ii) with an attenuated DENV strain (intertypic chimera). The vaccine where chimera of DENV has been made with another flavivirus is the chimeric yellow fever-dengue (CYD) vaccine, which is being developed by Sanofi Pasteur and licensed under the brand name "Dengvaxia" [18]. In this vaccine, prM and E genes of the attenuated yellow fever LAV strain 17D have been replaced with the corresponding genes from DENV [19]. The validation behind this design was the fact that humoral response against the structural proteins of dengue was responsible for protective immunity during natural contagion and thus these chimeras would generate a protective immune response in vaccines. A tetravalent mixture of the four chimeric viruses has undergone extensive clinical evaluation and has recently been approved in Mexico, Brazil, El Salvador, and Philippines [20]. This vaccine will be discussed in detail in the later sections.

It was observed that DENV-2 was the dominating component and its replicative potential reduced by increasing the DENV-3 and DENV-4 component. This variation in DENV-2 induced viremia due to the variation in the dose of DENV-3 and DENV-4 components indicated viral interference. Moreover, the neutralizing antibody titers were found to be significantly low against DENV-4 and despite this, macaques were found to be protected against DENV-4 challenge [21]. A phase I clinical trial of low and high doses of the DENVax in healthy subjects in Columbia revealed that the candidate was safe and immunogenic. Notably, it corroborated the findings made in nonhuman primate study that neutralizing 
Oviya et al. / GSC Biological and Pharmaceutical Sciences 2019, 06(03), 016-023

antibody titers elicited by DENVax are lowest against DENV-4 and highest against DENV-2 [22]. Additional insights into its effectiveness will be revealed through its phase II clinical trial evaluation. Meanwhile, phase III evaluation of this vaccine has now been started [23].

\subsection{Nonreplicating viral vaccines}

These vaccine candidates are not capable of replicating and thus offer the advantage of conferring immunity without the risk of infection. There are multiple strategies to develop this class of vaccines like DNA vaccines, subunit proteins, VLPs, and so forth:

Advantages: reduced reactogenicity, better suitability for immune-compromised individuals, and balanced immune response in case of tetravalent formulation.

Disadvantages: less broad, potent, and durable immune response, which may result in ADE, and requiring the use of adjuvants.

Recombinant antigens based on DENV EDIII have been produced by different groups using E. coli and yeast expression hosts. Recombinant EDIII antigens, expressed either independently or fused to different carriers such as maltose binding protein [24] and the Neisseria meningitides p64k protein, have been shown to generate anti-DENV immune responses in mice and nonhuman primates [25-27]. These vaccines candidates are in preclinical phase currently.

\subsection{Dengue DNA vaccine}

This antibody comprises of a plasmid vector containing the gene(s) encoding for an antigen, which on inoculation is taken up by antigen introducing cells (APCs). When the plasmid enters the cell, it codes for the antigen which at last gets related with MHC class I atoms and gets showed on the cell surface, prompting defensive cytotoxic invulnerable reaction. Maritime Medical Research Center (NMRC), USA, has built up a DENV-1 DNA antibody competitor (D1ME100) by cloning prM and E quality of DENV-1 serotype into plasmid vector, which was broadly assessed in mice and macaques without adjuvant [28] before stage 1 preliminaries in solid grown-ups. In spite of the fact that DENV-1 DNA immunization was observed to be all around endured, the killing counter acting agent titers and the quantity of responders were observed to be low $[28,29]$. Along these lines, to upgrade its immunogenicity, a lipid-based adjuvant Vaxfectin was investigated. Tetravalent dengue DNA antibody (TVDV) was assessed for immunogenicity with and without Vaxfectin in macaques. It was seen that Vaxfectin brought about higher and increasingly steady (assessed till a half year after definite lift) titers. The normal balance titers with TVDV/V axfectin against DENV-1, DENV-2, DENV-3, and DENV-4 every month after conclusive lift were roughly 200, 270,170, and 70, individually. A half year after the last lift, the titers against DENV-2 and DENV-3 decreased, while those against DENV-1 and DENV-4 expanded hardly. In the gathering without Vaxfectin, titers against DENV-2 just were perceivable a half year after the last lift. Additionally, Vaxfectin permitted better security from viremia against DENV-2 challenge [30]. In the wake of setting up nontoxicity of TVDV/Vaxfectin in New Zealand white rabbits [31], a stage I preliminary was started in 2011 in USA [32].

\subsection{Replication-defective virus vectored vaccines}

In this method, a virus is used as a vector to carry antigenic genes that are capable of eliciting neutralizing antibody response. A few instances of viral vectors are adenovirus vectors, Venezuelan equine encephalitis virus vector, and attenuated measles virus [33-35]. A case of virus vectored dengue vaccine is cAdVax. It consists of bivalent constructs expressing prM and E proteins from two dengue serotypes each (DENV-1 and DENV-3 together in one and DENV-2 and DENV-4 in another construct). Study in NHPs showed production of neutralizing antibodies to respective DENV serotypes [36]. Therefore, a tetravalent formulation (cAdVax-DenTV) was prepared by mixing the bivalent constructs, which showed protection against all serotypes on DENV challenge in Rhesus macaques [37].

\subsection{Virus like particle (VLP) vaccines}

The prM and E proteins of DENVs co expressed in heterologous hosts have been shown to co assemble into VLPs. Thus, a vaccine based on physical mixtures of four monovalent DENV VLPs can be developed to have a tetravalent formulation [38]. From the perception of using VLPs for vaccine purpose, the yeast system may be more suitable, as it has the potential for higher yields and can glycosylate the antigens. Recent work indicates that yeast expressed-DENV E ectodomain forms VLPs in the absence of prM [39]. Another approach is based on displaying the DENV EDIII on VLPs formed by hepatitis B virus core antigens [40]. 


\section{Conclusion}

The improvement of severe dengue disease is a multifactorial process in which the presence of preexisting heterologous antibodies plays an important role. The absolute need for an efficacious tetravalent DENV vaccine, lack of an adequate animal disease model, and immune correlates of diseases protection remain as some of the major obstacles in developing a successful dengue vaccine. Since the wild type mice do not replicate clinical signs of human dengue infection, genetically engineered mouse models have been developed with considerable success to mimic some aspects of human infection. The most successful system has been the use of mouse-adapted DENV-2 and AG129 mice that lack IFN $-\alpha \beta \gamma$ receptors. Due to suppression of IFN pathway, an important branch of host immune response is disabled, which allows DENV to replicate. AG129 mice on disease with mouse-adjusted DENV-2 create vascular spillage without neurological entanglements, in this way copying human clinical indications of serious dengue. Additionally, this mouse show has been observed to be valuable in scoring ADE by inactive exchange of against DENV antibodies and test with nonlethal portion of mouse-adjusted DENV-2. The inactively exchanged antibodies are said to upgrade the ailment if the mice capitulate to disease and pass on. Since mouse-adjusted DENVs are not the normally circling strains, AG129 mice are being investigated as a reasonable dengue demonstrate with clinical isolates. Resistant reactions evoked by most dengue immunization approaches dependent in general infection might be like regular DENV contaminations and in this manner ailment or safe improvement methodologies (prevalent serotype cross-receptive killing antibodies) of DENV may defeat the defensive (minor serotype specific killing antibodies) viability of the entire infection based antibody competitor. A viable dengue immunization must be planned, which is equipped for inspiring dominatingly DENV serotype-explicit killing (defensive) antibodies without serotype cross-responsive killing (disease enhancing) antibodies. The pipeline of dengue antibodies is developing and despite lower adequacy, a dengue immunization may before long have turned out to be accessible for human use. Continued research on this topic is required to fill important gaps in our knowledge in the immune pathogenesis of severe dengue disease and may guide the development of safe and efficacious antiviral drugs and vaccines.

\section{Compliance with ethical standards}

\section{Acknowledgments}

I gladly acknowledge to the Head of Department, Department of Microbiology, Annamalai University, Faisalabad for wholly contribution and providing all the needs in review work.

\section{Disclosure of Conflict of interest.}

We have no conflicts of interest to disclose.

\section{References}

[1] Giri S and Singh AK. (2014). Assessment of surface water quality using heavy metal pollution index in Subarnarekha River, India. Water Quality, Exposure and Health, 5(4), 173-182.

[2] Murphy BR and Whitehead SS. (2011). Immune response to dengue virus and prospects for a vaccine. Annual review of immunology, 29, 587-619.

[3] Gubler DJ. (2006). Dengue/dengue haemorrhagic fever: history and current status. In New Treatment Strategies for Dengue and Other Flaviviral Diseases: Novartis Foundation Symposium, 277, 3-22.

[4] World Health Organization, Special Programme for Research, Training in Tropical Diseases, World Health Organization. Department of Control of Neglected Tropical Diseases, World Health Organization. Epidemic, \& Pandemic Alert. (2009). Dengue: guidelines for diagnosis, treatment, prevention and control. World Health Organization.

[5] Bhatt S, Gething PW, Brady OJ, Messina JP, Farlow AW, Moyes CL and Myers MF. (2013). The global distribution and burden of dengue. Nature, 496(7446), 504.

[6] Malavige GN, Fernando S, Fernando DJ and Seneviratne SL. (2004). Dengue viral infections. Postgraduate medical journal, 80(948), 588-601.

[7] Stanaway JD, Shepard DS, Undurraga EA, Halasa YA, Coffeng, LE, Brady OJ and Chuang TW. (2016). The global burden of dengue: an analysis from the Global Burden of Disease Study 2013. The Lancet infectious diseases, 16(6), 712-723. 
[8] World Health Organization, Special Programme for Research, Training in Tropical Diseases, World Health Organization. Department of Control of Neglected Tropical Diseases, World Health Organization. Epidemic, \& Pandemic Alert. (2009). Dengue: guidelines for diagnosis, treatment, prevention and control. World Health Organization.

[9] Bhatt S, Gething PW, Brady OJ, Messina JP, Farlow AW, Moyes CL and Myers MF. (2013). The global distribution and burden of dengue. Nature, 496(7446), 504.

[10] Brady OJ, Gething PW, Bhatt S, Messina JP, Brownstein JS, Hoen AG and Hay SI. (2012). Refining the global spatial limits of dengue virus transmission by evidence-based consensus. PLoS neglected tropical diseases, 6(8), e1760.

[11] Malavige GN, Fernando S, Fernando DJ and Seneviratne SL. (2004). Dengue viral infections. Postgraduate medical journal, 80(948), 588-601.

[12] Stanaway JD, Shepard DS, Undurraga EA, Halasa YA, Coffeng LE, Brady OJ and Chuang T.W. (2016). The global burden of dengue: an analysis from the Global Burden of Disease Study 2013. The Lancet infectious diseases, 16(6), 712-723.

[13] Ackermann M and Padmanabhan R. (2001). De novo synthesis of RNA by the dengue virus RNA-dependent RNA polymerase exhibits temperature dependence at the initiation but not elongation phase. Journal of Biological Chemistry, 276(43), 39926-39937.

[14] Modis Y, Ogata S, Clements D and Harrison SC. (2005). Variable surface epitopes in the crystal structure of dengue virus type 3 envelope glycoprotein. Journal of virology, 79(2), 1223-1231.

[15] Swaminathan S and Khanna N. (2013). Experimental dengue vaccines. In Molecular Vaccines, 135-151, Springer, Vienna.

[16] Barniol J, Gaczkowski R, Barbato EV da Cunha RV, Salgado D, Martínez E and Lum, LC. (2011). Usefulness and applicability of the revised dengue case classification by disease: multi-centre study in 18 countries. BMC infectious diseases, 11(1), 106.

[17] Narvaez F, Gutierrez G, Pérez MA, Elizondo D, Nuñez A, Balmaseda A and Harris E. (2011). Evaluation of the traditional and revised WHO classifications of Dengue disease severity. PLoS neglected tropical diseases, 5(11), e1397.

[18] De Alwis R, Smith SA, Olivarez NP, Messer WB, Huynh JP, Wahala WM and De Silva AM. (2012). Identification of human neutralizing antibodies that bind to complex epitopes on dengue virions. Proceedings of the National Academy of Sciences, 109(19), 7439-7444.

[19] Guirakhoo F, Weltzin R, Chambers TJ, Zhang ZX, Soike K, Ratterree M and Monath TP. (2000). Recombinant chimeric yellow fever-dengue type 2 virus is immunogenic and protective in nonhuman primates. Journal of virology, 74(12), 5477-5485.

[20] Guy B, Saville M and Lang J. (2010). Development of Sanofi Pasteur tetravalent dengue vaccine. Human vaccines, 6(9), 696-705.

[21] Osorio JE, Brewoo JN, Silengo SJ, Arguello J, Moldovan IR, Tary-Lehmann M and Stinchcomb DT. (2011). Efficacy of a tetravalent chimeric dengue vaccine (DENVax) in Cynomolgus macaques. The American journal of tropical medicine and hygiene, 84(6), 978-987.

[22] Osorio JE, Velez ID, Thomson C, Lopez L, Jimenez A, Haller AA and Luy BE. (2014). Safety and immunogenicity of a recombinant live attenuated tetravalent dengue vaccine (DENVax) in flavivirus-naive healthy adults in Colombia: a randomised, placebo-controlled, phase 1 study. The Lancet Infectious Diseases, 14(9), 830-838.

[23] Khetarpal N and Khanna I. (2016). Dengue fever: causes, complications, and vaccine strategies. Journal of immunology research, 2016.

[24] Simmons M, Murphy GS and Hayes CG. (2001). Antibody responses of mice immunized with a tetravalent dengue recombinant protein subunit vaccine. The American journal of tropical medicine and hygiene, 65(2), 159-161.

[25] Hermida L, Rodríguez R, Lazo L, Bernardo L, Silva R, Zulueta A and Guillén G. (2004). A fragment of the envelope protein from dengue-1 virus, fused in two different sites of the meningococcal P64k protein carrier, induces a functional immune response in mice. Biotechnology and applied biochemistry, 39(1), 107-114.

[26] Hermida L, Rodriguez R, Lazo, L, Silva, R, Zulueta, A, Chinea, G and Guillén G. (2004). A dengue-2 Envelope fragment inserted within the structure of the P64k meningococcal protein carrier enables a functional immune response against the virus in mice. Journal of virological methods, 115(1), 41-49. 
[27] Hermida L, BernardonL, Martín J, Alvarez M, Prado I, López C and Pérez AB. (2006). A recombinant fusion protein containing the domain III of the dengue-2 envelope protein is immunogenic and protective in nonhuman primates. Vaccine, 24(16), 3165-3171.

[28] Danko JR, Beckett CG and Porter KR. (2011). Development of dengue DNA vaccines. Vaccine, 29(42), 7261-7266.

[29] Beckett CG, Tjaden J, Burgess T, Danko JR, Tamminga C, Simmons M and Hayes CG. (2011). Evaluation of a prototype dengue-1 DNA vaccine in a Phase 1 clinical trial. Vaccine, 29(5), 960-968.

[30] Porter KR, Ewing D, Chen L, Wu SJ, Hayes CG, Ferrari M and Raviprakash K. (2012). Immunogenicity and protective efficacy of a vaxfectin-adjuvanted tetravalent dengue DNA vaccine. Vaccine, 30(2), 336-341.

[31] Raviprakash K, Luke T, Doukas J, Danko J, Porter K, Burgess T and Kochel T. (2012). A dengue DNA vaccine formulated with Vaxfectin ${ }^{\circ}$ is well tolerated, and elicits strong neutralizing antibody responses to all four dengue serotypes in New Zealand white rabbits. Human vaccines \& immunotherapeutics, 8(12), 1764-1768.

[32] Khetarpal N, and Khanna I. (2016). Dengue fever: causes, complications, and vaccine strategies. Journal of immunology research, 2016.

[33] Raviprakash K, Wang D, Ewing D, Holman DH, Block K, Woraratanadharm J and Porter K. (2008). A tetravalent dengue vaccine based on a complex adenovirus vector provides significant protection in rhesus monkeys against all four serotypes of dengue virus. Journal of virology, 82(14), 6927-6934.

[34] White LJ, Parsons MM, Whitmore AC, Williams BM, De Silva A and Johnston RE. (2007). An immunogenic and protective alphavirus replicon particle-based dengue vaccine overcomes maternal antibody interference in weanling mice. Journal of virology, 81(19), 10329-10339.

[35] Brandler S, Lucas-Hourani M, Moris A, Frenkiel MP, Combredet C, Février M and Tangy F. (2007). Pediatric measles vaccine expressing a dengue antigen induces durable serotype-specific neutralizing antibodies to dengue virus. PLoS neglected tropical diseases, 1(3), e96.

[36] Holman DH, Wang D, Raviprakash K, Raja NU, Luo M, Zhang J and Dong JY. (2007). Two complex, adenovirusbased vaccines that together induce immune responses to all four dengue virus serotypes. Clinical and Vaccine Immunology, 14(2), 182-189.

[37] Raja NU, Holman DH., Wang D, Raviprakash, K, Juompan, LY, Deitz, SB and Dong JY. (2007). Induction of bivalent immune responses by expression of dengue virus type 1 and type 2 antigens from a single complex adenoviral vector. The American journal of tropical medicine and hygiene, 76(4), 743-751.

[38] Suzuki R, Winkelmann, ER. and Mason PW. (2009). Construction and characterization of a single-cycle chimeric flavivirus vaccine candidate that protects mice against lethal challenge with dengue virus type 2. Journal of virology, 83(4), 1870-1880.

[39] Mani S, Tripathi L, Raut R, Tyagi P, Arora U, Barman T and Swaminathan S. (2013). Pichia pastoris-expressed dengue 2 envelope forms virus-like particles without pre-membrane protein and induces high titer neutralizing antibodies. PLoS One, 8(5), e64595.

[40] Swaminathan S and Khanna N. (2013). Experimental dengue vaccines. In Molecular Vaccines (pp. 135-151). Springer, Vienna.

\section{How to cite this article}

Oviya S, Kaviya S and Udhaya S. (2019). Dengue fever: Causes, complications, and vaccine strategies - A review. GSC Biological and Pharmaceutical Sciences, 6(3), 16-23. 